\title{
Energy Efficient Detections of Critical Events in Wireless Sensor Networks
}

\author{
Yunhuai $\mathrm{Liu}^{\dagger}$, Yanmin $\mathrm{Zhu}^{*}$, and Lionel $\mathrm{Ni}^{\dagger}$ \\ ${ }^{\dagger}$ Computer Science and Engineering, HKUST, Hong Kong \\ *Imperial College London, United Kingdom
}

\begin{abstract}
In many mission critical applications, one of the central tasks is to obtain aggregate values, such as AVERAGE and SUM, based on which critical conditions can be detected. Immediate measures can subsequently be taken to remove associated risks timely. However, there is no successful work for detecting such critical events energy efficiently. In this paper we propose a novel approach. A fully distributed algorithm is designed, in which each sensor makes the decision purely locally. The elegance of the algorithm is that a global network behavior can be presented based on individual node actions, without a central authority to coordinate. This effectively reduces unnecessary sensor reports for network-wide coordination. Our proposed algorithm, called MEES, has the property of a zero event-missing rate, i.e., critical events are always detected. There is a tradeoff between the energy cost and the false positive. Simulation experimental results show that MEES is more appropriate for large-scale WSNs with highly dynamic sensing environments. The energy cost can be saved up to $70 \%$.
\end{abstract}

\section{INTRODUCTION}

For a wide spectrum of applications ranging from habitat monitoring [1] to battlefield surveillance, the wireless sensor network (WSN) technology [2] has exhibited revolutionary advantages when compared to traditional solutions. Such advantages include low cost, easy deployment, fidelity sensing, self-organization, among the others. However, severe resource constraint is one of the most important characteristics of WSNs, which makes efficient design highly necessary. In particular, conserving the limited power on sensors has been a major task for WSN designs.

In this paper we investigate the challenges in sensor network design for mission-critical applications such as mine tunnels monitoring. In such applications, one or more sensory measurements are continuously monitored. When the measurement exceeds a given threshold, it would become critical and serious consequences may follow. For example, when the gas concentration in the mine tunnels is larger than a certain threshold, an explosion may happen, which could cause serious fatalities and huge property loss. Therefore, upon perception of such a critical event, an immediate response must be taken to remove this critical condition. For instance, the fans should be switched on to increase air circulation when the gas intensity is too high, and the explosion risk can be safely removed.

The central problem for these applications is to obtain a global aggregated value, e.g., the average value of gas concentration in mine tunnels monitoring or the sum of vehicles in a congested road. Note that the global property is necessary since individual sensors only provide partial information over a small area. In addition, individual readings may be inaccurate or even odd due to hardware faults. A straightforward solution to this problem is that every sensor reports its reading back to the central gateway. A central server computes the global aggregated value, based on which the action decision can be made. This is, however, a costly solution in large-scale and highly dynamic environment. A sensor reading may have to undergo multi-hop routing before it arrives at the gateway. Recent study has revealed that wireless communication is the main source of power consumption of sensors. Thus, this straightforward solution is energy inefficient and hence impractical for real-world applications. As a result, the key issue here is to detect critical events that the global average exceeds a predefined threshold in an energy efficient way.

The energy inefficiency of the centralized scheme has motivated many recent studies [3], [4], [5], [6], [7], [8], [9] on efficient computation of aggregate information, such as COUNT, SUM, AVERAGE, MAX and MIN, from a sensor network. Their central idea is to distribute the computation from the central unit to across the sensor network (i.e., in-network data processing). By this means, the data are processed as early as possible and the volume of data to be transmitted is therefore reduced. The studies can be generally divided into two categories. One category [3], [8], [9] computes exact aggregate values, and the other [4], [5], [6], [7], [10] computes approximate aggregate values.

Although these studies contribute to significant energy savings and provide useful aggregate information, they are not suitable for mission-critical applications. Several reasons account for the unsuitability. First, the existing works assume that the process of computing an aggregate is driven by an user query. However, critical events arise spontaneously. The users have no means to predict such events and it is impossible for them to issue a query beforehand. Periodic queries appear to be a solution to this problem. But, critical events have the rare nature. Periodic queries incur huge and unnecessary wastes of power. Second, aggregation algorithms [3], [8], [9] for computing exact aggregate information may work well in a static environment. In highly dynamic environments, however, aggregation algorithms can hardly take the advantage of data aggregations. Sensor reading may vary frequently in an unsynchronized manner. Note that tiny variation in individual nodes may result in a huge change of the global aggregates, and thus such tiny changes have to be always reported. Third, approximate aggregates provided by the approximation algorithms [4], [5], [6], [7], [10] are not accurate. Consequently, 
using these approximate values may result in missing of critical events.

In these decision-making schemes, the decision is made by a single authority (e.g., the sink or actuator) according to the global network property. Network-wide communications are necessary to gather information from each network part regardless the aggregation is applied or not. Such networkwide communications are one of the main energy consumption sources. To deal with this problem, we propose a novel approach by taking the rare nature of critical events into account. A distributed algorithm is developed. The main challenge in the algorithm design is conversion from the global property to a local condition. More specifically, what strategy each sensor node shall take, with only local information, so that the global network property is guaranteed. Note that we are serving for mission critical applications. Approximated values without bound do not suffice due to the possible false positive. Other-wise, critical events may not be detected, causing severe con-sequences.

In our approach, called MEES, each sensor maintains not only its own readings but also a local average over its certain neighborhood. It is only when the local average exceeds the given threshold that the sensor reports its readings to the gateway. We prove that for each sensor, when its local average is smaller than the threshold, it can safely keep silent for the energy saving purpose. A report of high reading received at the gateway indicates that a certain area produces consistent high sensor reading. In real-world environments, such a consistent view in a certain area is a strong indicator that the global aggregate is also over the threshold. Thus, an action of polling all sensors can be triggered to obtain the accurate aggregate. Using a local average instead of a single reading can dramatically reduce the chance of unnecessary reports of a sensor. The rational in behind is that a high sensor reading can be effectively offset by other low readings in the neighbor-hood.

The rest of the paper is structured as follows. The next section covers related work. In Section III, we present our solution in details. Section IV describes the evaluation methodology and presents performance evaluation results. Finally, we conclude the paper by drawing conclusions and discussing future work in Section V.

\section{RELATED WORK}

In large-scale sensor networks, aggregate information is more useful than individual sensor readings [3]. It is aggregate information that helps human users to make reliable decisions regarding the target monitored by a sensor network. The centralized scheme to compute aggregates suffers poor energy efficiency. Thus, it is excluded from practical use. This necessitates the design of efficient aggregation algorithms.

In TinyDB [9], a sensor network is viewed as a distributed sensor database and queries are support to extract desired data from the network. Aggregate queries are concerned with aggregate information instead of individual sensor readings. Efficient in-network processing of aggregate queries has been the subject of extensive research.
Several algorithms [3], [8], [9], [11] are able to compute exact aggregate values. They usually rely on an underlying tree-based routing. For example, Tiny Aggregation (TAG) [3] establishes a tree rooted at the sink when flooding the query. Extracted information is propagated from leaf nodes upwards to the sink. The advantage of tree-based aggregation algorithms is that value duplicates can be avoided. Some aggregates are duplicate insensitive, e.g., MAX and MIN. Other aggregates are duplicate sensitive, such as COUNT, SUM and AVERAGE. Tree-based algorithms can easily compute duplicate sensitive aggregates without additional control because each value is used only once. However, a tree routing is prone to affection by node or communication failures. When a sensor fails, the information contained in the whole branch led by the node is lost.

To be robust against node and communication failures, some algorithms [5], [6], seek to exploit multi-path routing. Nevertheless, an issue is consequently raised that duplicate sensitive aggregates become difficult to compute. One important solution is to use duplication-insensitive synopses [6]. A rings routing structure that supports multi-path routing is used for propagating synopsis in [6]. In many other algorithms [4], [7], [10], [11], a simpler routing scheme called random gossip is employed. By gossip, a sensor only exchanges information with its neighbors. Synopsis can be diffused across the network after a number of rounds. It is guaranteed that the estimated aggregate value at each sensor is close to the real one. For these gossip-based algorithms, the convergence speed is the one of the most important performance metrics [4]. Although synopsis is a powerful tool for aggregation computation, the resulting aggregates are not accurate and embrace a certain error.

These aforementioned algorithms greatly improve energy efficiency for aggregation computation in sensor networks. However, they cannot accommodate mission critical applications studied in this paper. The reasons have been studied in Section I.

Routing is a fundamental building block for sensor networks. This paper does not consider the routing issue. A lot of efficient routing algorithms [12], [13], [14], [15], [16], however, have been proposed and can be incorporated in different applications. Depending on different needs, various routing algorithms can be selected.

The direct diffusion [12] establishes a routing path by inverting the path followed by a discovery packet. In two-tier routing [13], a grid structure is built and maintained for routing data. Normal sensors first deliver its data to a sensor on the grid structure and then the data will be routed on the structure. When the location information is available, geographic routing can be exploited. In GPSR [14], a greedy routing strategy is proposed by which a sensor always chooses the neighbor closest to the destination as the next hop. Ye et al. designed a hop count based routing scheme [15]. Every sensor in the network is aware of its distance away from the sink in terms of hop counts. To routing a packet, a sensor forwards the packet to one neighbor having a short hop distance. Rumor routing is a simple algorithm, in which a sensor forwards packets to a random neighbor. No state information is required, which 
makes this routing algorithm very scalable.

\section{Measurement Estimation Scheme}

In this section, we present the design of the MEasurement Estimation Scheme (MEES). We first describe the adopted network model and assumptions we made in this work. We then give a naive approach to solve the problem. In the third part we derive some observations based on which we design MEES. In the fourth part, we give a detailed description of the scheme and in the last subsection we present the desired features and properties of MEES.

\section{A. Assumptions and network model}

We model a network using a direct graph $G(V, E)$, where $V$ is the node set and $E$ is the link set. To simplify the presentation, we assume the disk model. We will show, however, our scheme does not rely on this strong assumption. Every node $\mathrm{u}$ has a transmission range $T_{r}$. All other nodes within this range are neighbors of $u$. Let $\operatorname{Nr}(\mathrm{u})$ denote the set of neighboring nodes of $u$ plus the node $u$ itself, i.e. $N r(u)=\{v \mid(u, v) \in E\} \cup\{u\}$. Similarly, we use $N r_{k}(u)$ to denote $u$ 's k-hop neighbor set (actually $N r(u)=N r_{1}(u)$ and $\left.N r_{0}(u)=\{u\}\right)$. We assume a bi-directional neighbor relation, $v \in N r_{k}(u) \Leftrightarrow u \in N r_{k}(v)$. We assume a reliable network environment that there is no packet loss. This can be achieved by simple acknowledgement and retransmission techniques.

Suppose each node $u$ has a local measurement value $\zeta(u)$ which is obtained by $u$ 's sensing unit and dependent on applications. For example, in coal mine applications, $\zeta$ is the measured oxygen intensity. In moving object tracking and counting applications, it is the counted number of the target objects. Let $\bar{\zeta}_{G}$ denote the average of $\zeta$ over all the nodes in $G$

$$
\bar{\zeta}_{G}=\frac{\sum_{u \in V} \zeta(u)}{|V|}
$$

where $|\mathrm{V}|$ is the size of the node set. Note that as long as the network size $|\mathrm{V}|$ is available, $\bar{\zeta}_{G}$ can also be used to compute the total sum of the sensing measurements.

Let $\zeta_{T H}$ and $\zeta_{t h}$ denote two application-dependent thresholds of $\bar{\zeta}_{G}$. $\zeta_{T H}$ is the upper threshold and $\zeta_{t h}$ is the lower one. In the normal cases, the global average $\bar{\zeta}_{G}$ is within this range, i.e., $\bar{\zeta}_{G} \in\left[\zeta_{t h}, \zeta_{T H}\right]$. We call $\left[\zeta_{t h}, \zeta_{T H}\right]$ the validated domain. We define,

Definition: A critical event occurs when the change of a certain node's sensing measurement invalidates the global constraint of $\bar{\zeta}_{G} \in\left[\zeta_{t h}, \zeta_{T H}\right]$

We assume the validated domain is available in each node, which can be obtained by a simple network broadcast in the initialization and upon updates. The objective of MEES is to identify all critical events (i.e., zero missing rate). When critical events happen, responding actions shall be taken immediately to prevent the potential or further property loss and severe casualties. In these scenarios, a zero missing rate is critical.

We assume a highly dynamic environment with unsynchronized measurement varying patterns. In such environments, the sensing measurements $\zeta(\mathrm{u})$ vary largely and frequently, depending on application specifications. More over, different nodes will have varied sensing measurements at different time in an unsynchronized manner.

\section{B. A naive approach}

A naive approach can be based on measurement update mechanism. Upon sensing a different sensing measurement, a node shall report the value to certain data aggregation node (e.g., the sink, a cluster head or actuator). Once all values are available in the aggregation node, it can easily compute the global average by equ. (1). It then notifies other nodes by a network-wide broadcast.

In a static sensing environment, this scheme may work well. Aggregation techniques can also be used to reduce the energy cost of information gathering. By aggregation, the in-formation retrieval is very efficient. Ideally the worst energy cost is $2|\mathrm{~N}|$, i.e., each node exactly transmits twice, one for report and one for broadcast.

In a dynamic environment, however, sensing measurements may vary frequently. When a node senses a different measurement, it has no knowledge whether other nodes also have different values. Thus, it does not know whether its new value will make the global average to fall outside the validated domain $\left[\zeta_{t h} \zeta_{T H}\right]$. In order to guarantee no event report is missed, every node, upon sensing a different measurement, has to report to the aggregation node immediately. As other nodes may not have varied sensing measurement then, aggregation techniques take no advantage. In the analysis section, we will see in average how much cost will be paid to guarantee a zero missing rate of critical events.

\section{Preliminary}

The naive approach verifies the constraint by computing the accurate $\bar{\zeta}_{G}$. This is, however, not always necessary in our application scenarios. The observation is that we can safely stop the computation when we are sure $\bar{\zeta}_{G}$ is within the validated domain $\left[\zeta_{t h}, \zeta_{T H}\right]$. If we know that $\bar{\zeta}_{G}$ is bounded in $\left[\zeta_{t h}, \zeta_{T H}\right]$, no actual value of $\bar{\zeta}_{G}$ is needed. In addition, an efficient algorithm shall be localized and fully distributed so that no costly network-wide communication is required

Another observation is that sensor nodes are working in a cooperative environment. From each node's perspective, all other nodes are trusted and dependable. If it takes certain strategy to follow, it can assume other nodes will follow the same strategy as well. From the whole network's perspective, a global network behavior will be presented when individual nodes share the philosophy. The key issue is what individual actions can result in a desired global behavior.

Instead of computing the accurate value of $\bar{\zeta}_{G}$, in MEES we let each node obtain an approximated bound of the global average $\bar{\zeta}_{G}$. Towards this end, we explore following theorems and lemmas.

Theorem 3.1: : The global average $\bar{\zeta}_{G}$ is no more than the maximal, and no less than the minimal of $\zeta(\mathrm{u})$ over all nodes in the network, i.e.,

$$
\min \{\zeta(u), u \in V\} \leq \bar{\zeta}_{G} \leq \max \{\zeta(u), u \in V\} .
$$




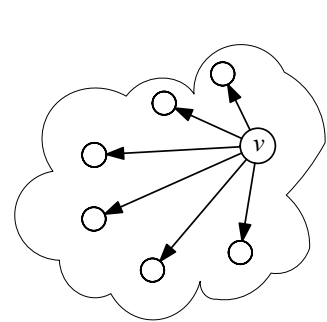

(a). a hub network

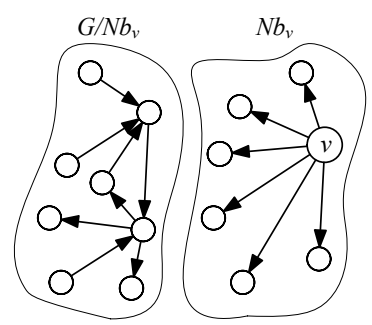

(b). a general network
Fig. 1. An example of hub graph

Equalities hold when all nodes have the same $\zeta(\mathrm{u})$.

Proof : This can be easily proved by contradiction. We omit the proof due to the simplicity.

Given an arbitrary graph $G(V, E)$, If $G$ contains a node $v_{0}$ that has links to every other node, then $N r\left(v_{0}\right)=G$ and thus $\bar{\zeta}_{N r\left(v_{0}\right)}=\bar{\zeta}_{G}$. We call such a graph hub graph. An example of a hub graph is illustrated in 1 (a).

Lemma 3.2: $\bar{\zeta}_{G} \geq \min \left(\bar{\zeta}_{N r(v)}\right)$ if $G$ is a hub graph.

Proof We omit the proof due to the simplicity.

Theorem 3.3: : For any topology G, the global average $\bar{\zeta}_{G}$ is no more than the maximal and no less than the minimal of local average $\bar{\zeta}_{N r(u)}$ of all nodes in the network, i.e.,

$$
\min \left\{\bar{\zeta}_{N r(u)}\right\} \leq \bar{\zeta}_{G} \leq \max \left\{\bar{\zeta}_{N r(u)}\right\}
$$

where the local average $\bar{\zeta}_{N r(u)}$ can be computed by,

$$
\bar{\zeta}_{N r(u)}=\frac{\sum_{v \in N r(u)} \zeta(v)}{|N r(u)|}
$$

Proof We use mathematic induction on the network size $|V|$.

1) When $|V|=1, G$ is a hub graph. Accordingly to Lemma 3.2 , the statement stands.

2) Assume for any graph of size $|V| \leq k$, the statement stands. We state that for any graph $G$ of $|V|=k+1$, the statement stands. Select any node in $G$ denoted as $v_{0}$. Partition $G$ to two parts: one is $v_{0}$ 's neighboring set $N b_{v_{0}}$ (including $v_{0}$ ), and the other is $N b_{v_{0}}$ 's complement set $G \backslash N b_{v_{0}}$. An example of $N b_{v_{0}}$ and $G \backslash N b_{v_{0}}$ is illustrated in Fig. 1 (b). There can be three cases:

a). $N b_{v_{0}}=\left\{v_{0}\right\}$, and thus $\left|V\left(G \backslash N b_{v_{0}}\right)\right|=k$. Base on the assumption, $\exists v_{1} \in G \backslash N b_{v_{0}}, \lambda\left(G \backslash N b_{v_{0}}\right)>$ $\left(\operatorname{avg}\left(\lambda_{G \backslash N b_{v_{0}}}\left(v_{1}\right)\right)\right)$. Thus:

$$
\begin{aligned}
\lambda(G) & =\frac{\lambda\left(G \backslash N b_{v_{0}}\right) \cdot\left|G \backslash N b_{v_{0}}\right|+\lambda_{G}\left(v_{0}\right)}{\left|G \backslash N b_{v_{0}}\right|+1} \\
& \geq \min \left(\operatorname{avg}\left(\lambda_{G \backslash N b_{v_{0}}}\left(v_{1}\right), \lambda_{G}\left(v_{0}\right)\right)\right) \\
& \geq \min \left(\operatorname{avg}\left(\lambda_{G}(v)\right)\right)
\end{aligned}
$$

b). $N b_{v_{0}} \supset\left\{v_{0}\right\}$, and $N b_{v_{0}} \neq G$. In this case we have, $\left|V\left(G \backslash N b_{v_{0}}\right)\right|<k$. Thus:

$$
\exists v_{1} \in G \backslash N b_{v_{0}}, \lambda\left(G \backslash N b_{v_{0}}\right)>\operatorname{avg}\left(\lambda_{G}\left(v_{1}\right)\right)
$$

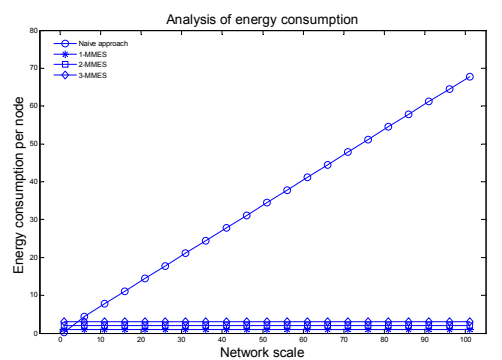

Fig. 2. Analytic results of the energy consumptions for the naive approach and $\mathrm{k}-\mathrm{MEES}$

As $N b_{v_{0}}$ is a hub-graph, we have:

$$
\lambda\left(N b_{v_{0}}\right)=\operatorname{avg}\left(\lambda_{G}\left(v_{0}\right)\right)
$$

Combining (2) and (3), we have:

$$
\begin{aligned}
\lambda(G)= & \frac{\lambda\left(G \backslash N b_{v_{0}}\right) \cdot\left|V\left(G \backslash N b_{v_{0}}\right)\right|}{|V(G)|} \\
& +\frac{\lambda\left(N b_{v_{0}}\right) \cdot\left|V\left(N b_{v_{0}}\right)\right|}{|V(G)|} \\
\geq & \min \left(\operatorname{avg}\left(\lambda_{G}\left(v_{1}\right)\right), \operatorname{avg}\left(\lambda_{G}\left(v_{0}\right)\right)\right) \\
\geq & \min \left(\lambda_{G}(v)\right)
\end{aligned}
$$

The statement stands.

c). $N b_{v_{0}}=G$. In this case, $G$ is a hub graph and thus the statement stands.

For all three cases, the statement stands.

\section{MEES design}

Theorem 2 ensures that $\bar{\zeta}_{G} \in\left[\min \left\{\bar{\zeta}_{N r(u)}\right\}, \max \left\{\bar{\zeta}_{N r(u)}\right\}\right]$. Thus if $\left[\min \left\{\bar{\zeta}_{N r(u)}\right\}, \max \left\{\bar{\zeta}_{N r(u)}\right\}\right] \subset\left[\zeta_{t h}, \zeta_{T H}\right]$ for $\forall u \in$ $G$, we can guarantee that $\bar{\zeta}_{G} \in\left[\zeta_{t h}, \zeta_{T H}\right]$. As such we make the global constraint $\bar{\zeta}_{G} \in\left[\zeta_{t h}, \zeta_{T H}\right]$ to be locally verifiable. The promising advantage of Theorem 2 is that the computation of $\left[\min \left\{\bar{\zeta}_{N r(u)}\right\}, \max \left\{\bar{\zeta}_{N r(u)}\right\}\right]$ only relies on the topology structure G. It is regardless of the geometric constraint. A pair of distant nodes can be virtual neighbors when they have a virtual link. By setting a number of virtual links, connect distant node pairs will also be neighbors. As such the measurement approximation bound $\left[\min \left\{\bar{\zeta}_{N r(u)}\right\}, \max \left\{\bar{\zeta}_{N r(u)}\right\}\right]$ can be tighter, though in the cost of addition energy consumption for communications between these distant virtual neighbors.

MEES works as follows. Initially the sink broadcasts the invalidated domain $\left[\zeta_{t h}, \zeta_{T H}\right]$ to the whole network. Each node $\mathrm{u}$ then communicates with its neighbor set $\mathrm{Nr}(\mathrm{u})$ to obtain their sensing measurements $\zeta(N r(u))$. If $\bar{\zeta}_{N r(u)}$ satisfies the condition $\bar{\zeta}_{N r(u)} \in\left[\zeta_{t h}, \zeta_{T H}\right]$, then u can safely stop until it senses a varied measurement $\zeta(u)$. Otherwise it uses a network-wide broadcast to invoke the naive approach. An extension of this basic MEES is applying an extended neighbor set $\mathrm{Nr}(\mathrm{u})$. Instead of using the directed neighbor set $\mathrm{Nr}(\mathrm{u}), \mathrm{u}$ can use its k-hop neighbors $N r_{k}(u)$ to verify the condition. That is, $u$ collects the sensing measurements of its k-hop neighbors $N r_{k}(u)$ and compute $\bar{\zeta}_{N r_{k}(u)}$. This extended MEES is denoted as $\mathrm{k}$-MEES, where $\mathrm{k}$ is a critical parameter to determine. 


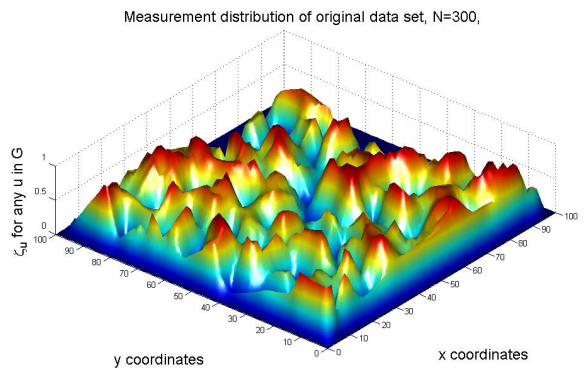

Fig. 3. Original sensory data of uniform distribution

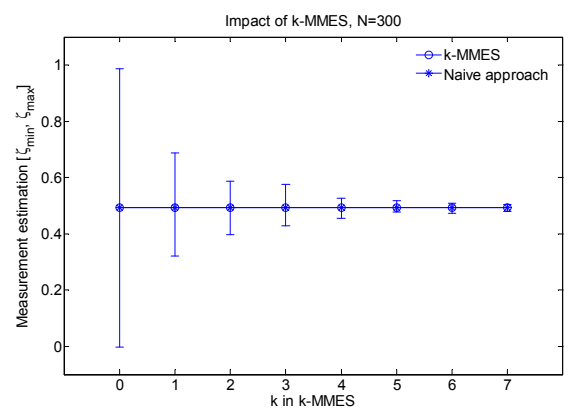

Fig. 5. $\left[\min \left\{\bar{\zeta}_{N r(u)}\right\}, \max \left\{\bar{\zeta}_{N r(u)}\right\}\right]$ in uniform distribution

\section{E. Analysis results}

In this section we give some analysis results of k-MEES. We first prove the correctness of k-MEES, and then analyze the energy saving when the sensor obtains a different measurement.

Theorem 3.4: : By k-MEES we have $\exists u \in V, \bar{\zeta}_{N r_{k}(u)} \notin$ $\left[\zeta_{t h}, \zeta_{T H}\right]$ if $\bar{\zeta}_{G} \notin\left[\zeta_{t h}, \zeta_{T H}\right]$.

Proof We omit the proof due to space limitations.

Theorem3.4 is critical to ensure the correctness of k-MEES. It guarantees a zero event missing rate. There will be no false positive error by k-MEES. When critical events happen, there must be a node giving a report to alert the aggregation node. If every node keeps silent, the system must be safe. On the contrary, false negative errors are possible. It is very possible that some nodes report the event, in which the aggregation nodes then issue the global query to check, and eventually find out that the system is still safe. Such false negative errors can be largely reduced by getting a tighter $\left[\min \left\{\bar{\zeta}_{N r(u)}\right\}, \max \left\{\bar{\zeta}_{N r(u)}\right\}\right]$ (e.g., increase k), in the cost of additional energy consumption. In the extreme case, when $\mathrm{k}$ is equal to the network diameter, k-MEES is the same as the naive approach. We will investigate the impact of $\mathrm{k}$ in the next performance evaluation section.

Next we give an analysis of the energy consumption for the naive approach and k-MEES upon a node sensing a different measurement. To simplify the analysis, we assume a diskshape application field centered at a single sink. The radius is $R$ hops. The density is d nodes per unit of area.

Assume the transmission is along a shortest hop path toward the sink in naive approaches. Each node $\mathrm{u}$ introduces $r(u)$ units of cost, where $r(u)$ is the hop distance from $\mathrm{u}$ to the

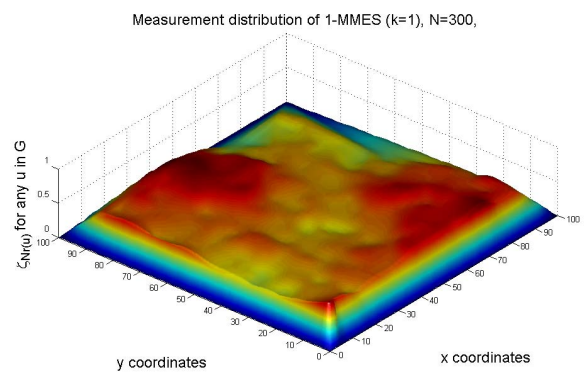

Fig. 4. 1-MEES data distribution of uniform distribution

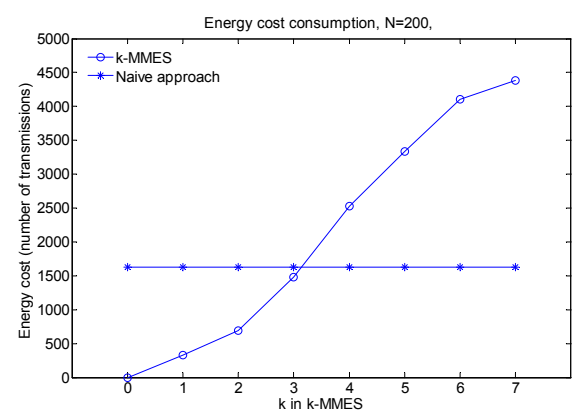

Fig. 6. Energy cost of naive approach and 1-MEES in uniform distribution

sink. The average cost is thus,

$$
\begin{aligned}
\bar{\varepsilon}_{\text {naive }} & =\frac{\sum_{u \in V} r(u)}{|V|}=\frac{\sum_{r=1}^{R} \pi r(2 r-1) d}{\pi R^{2} d} \\
& =\frac{\frac{1}{3} R(R+1)(2 R+1)-\frac{1}{2} R(R+1)}{R^{2}} \\
& =\frac{2}{3} R+\frac{1}{2}-\frac{1}{6 R}
\end{aligned}
$$

The average cost of k-MEES depends on the parameter $\mathrm{k}$. To obtain the k-hop neighbor information, in expectation a node needs $\mathrm{k}$ transmissions. The comparison of the naive approach cost and $\mathrm{k}-\mathrm{MEES}$ with different $\mathrm{k}$ is depicted in Fig. 2.

\section{Performance EVAluation}

In this section we evaluate the performance of k-MEES scheme. We conduct simulation experiments using Matlab. We first describe the experiment setup, and present the performance evaluation results. The sets of experiments are organized according to different data distributions.

\section{A. Experimental setup}

We assume a $100 \mathrm{~m} \times 100 \mathrm{~m}$ application field in which a number of sensor nodes are randomly deployed. We adopt the disk transmission model that there is an identical transmission range for each node. Within this range a pair of nodes can always communicate and beyond this range no one can communicate directly. For k-MEES, no specific routing protocol is required and the information is exchange using restricted broadcast within k-hop neighbors. For other measurement schemes, we assume a tree-based routing scheme is available to deliver information. The transmission range is set to be 10 


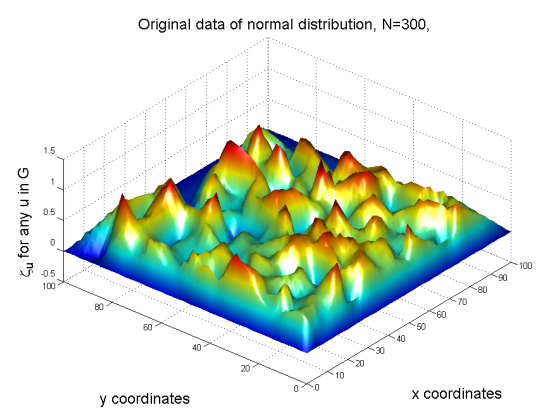

Fig. 7. Original sensory data of Gaussian distribution

meters, which is according to the large number of empirical studies. No congestions, collision, or radio interference is considered. Each data is averaged over 10 independent runs.

We assume that the sensory measurement domain is $[0,1]$, i.e., for each node $\mathrm{u}, \zeta(u) \in[0,1]$. This simplified model can be easily extended to other more realistic data domains. The performance of k-MEES is highly depending on the data distributions. Due to the space limitation we only show some representative ones. In this work we study three data distributions:

- Uniform data distribution: Many nature statistics follow this distribution. In this distribution, all validated numbers have the same probability to be chosen by a node. The probability density function is,

$$
f(x)=\left\{\begin{array}{l}
1,0 \leq x \leq 1 \\
0, x<0 \vee 1<x
\end{array}\right.
$$

Obviously the mean is 0.5 and the variance is $1 / 12$.

- Gaussian data distribution: It is also called normal distribution. It is a widely applied model for quantitative phenomena in the natural and behavioral sciences due to the central limit theorem. The probability density function is,

$$
\phi_{\mu, \sigma^{2}}=\frac{1}{\sigma \sqrt{2 \pi}} e^{-\frac{(x-\mu)^{2}}{2 \sigma^{2}}},
$$

where $\mu$ is the mean and $\sigma^{2}$ is the variance. In our experiments we set $\mu=0.5$ and $\sigma=0.5$

We compare k-MEES with the naive approach (Sec. 3.2) using two evaluation metrics,

- $\bar{\zeta}:$ In the naive and gossip-based approaches, the accurate $\bar{\zeta}$ is computed. In k-MMS, we show the derived upper and lower limit of $\bar{\zeta},\left[\min \left\{\bar{\zeta}_{N r(u)}\right\}, \max \left\{\bar{\zeta}_{N r(u)}\right\}\right]$. In practice, when $\left[\min \left\{\bar{\zeta}_{N r(u)}\right\}, \max \left\{\bar{\zeta}_{N r(u)}\right\}\right] \subset\left[\zeta_{t h}, \zeta_{T H}\right]$, it is guaranteed that there will be no critical events.

- $\varepsilon$ : The energy cost. This is to show how much energy will be consumed during the decision-making. In the naive approach, all data are transmitted back to the sink. In gossip-based algorithm, each sensor will deliver its data to all other nodes. In k-MEES, each sensor needs to deliver its data to k-hop neighbors $N r_{k}(u)$. Therefore gossip-based algorithms can be considered as a special case of $\mathrm{k}-\mathrm{MEES}$ with $\mathrm{k}$ equal to the network diameter.

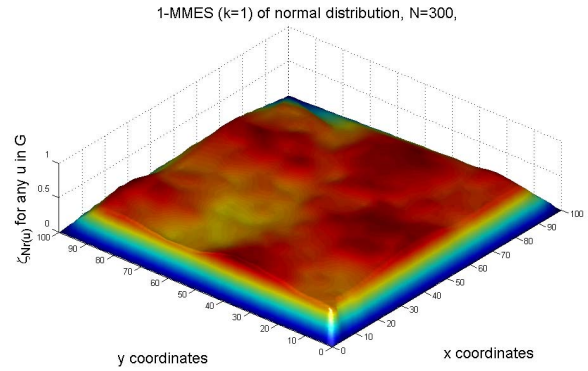

Fig. 8. 1-MEES data distribution of Gaussian distribution

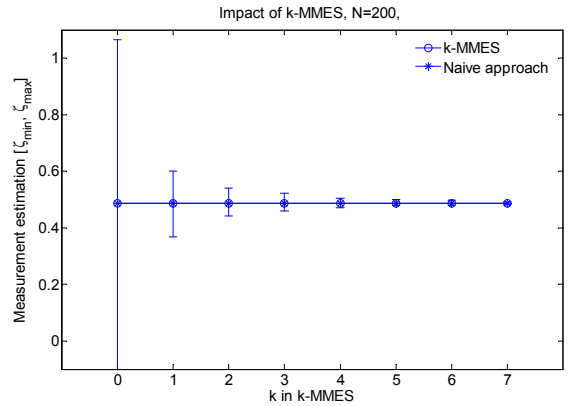

Fig. 9. $\left[\min \left\{\bar{\zeta}_{N r(u)}\right\}, \max \left\{\bar{\zeta}_{N r(u)}\right\}\right]$ in Gaussian distribution

\section{B. Uniform data distribution}

In the first set of experiments, we study the impact of the parameter $\mathrm{k}$ on the measurement estimation bound $\left[\min \left\{\bar{\zeta}_{N r(u)}\right\}, \max \left\{\bar{\zeta}_{N r(u)}\right\}\right]$ and the control communication overhead $\varepsilon$ under the uniform sensory data distribution. Fig. 3 and Fig. 4 give an example of the original and 1-MEES $(\mathrm{k}=1)$ data sets of the uniform distribution. 300 nodes are randomly uniformly deployed to an $100 \mathrm{~m} \times 100 \mathrm{~m}$ application field. The measurement estimation bound of k-MEES using different $\mathrm{k}$, from 0 to 8 , is depicted in Fig. 5. 0-MEES is actually the original data distribution. If we directly use this value to compute the bound, the deviation is about 0.5 , which is often useless in practice. 1-MEES significantly reduce the bound to $[0.35,0.65]$, one third of the original range. For applications of the threshold beyond this range, i.e., $[0.35,0.65] \subset\left[\zeta_{t h}, \zeta_{T H}\right]$, sensors can locally decide to keep silent. No global communication is needed. $\mathrm{k}=2, \mathrm{k}=3$ and $\mathrm{k}=4$ have the similar bounds. $\mathrm{k}>4$ shrink the bound to $[0.48,0.52]$, which is very close to the global value. In our setting, the network diameter is about 12 hops. naive approaches are regardless of the $\mathrm{K}$ and therefore the accurate $\bar{\zeta}$ is obtained under all circumstances.

A tighter range requires more energy cost. The cost of kMEES is nearly linear to the parameter k (Fig. 6). 0-MEES has no cost because every make decisions purely based on its own value. 1-MEES need exactly one transmit for each node, and therefore the cost is about 300. 2-MEES needs at most 2 transmits, while in some cases the information can be piggyback. Regardless of the parameter k, naive approaches always 
need about 1600 transmissions to collect all the information. When the network scales up, this cost will increase as well.

From this set of experiments, in uniform data distribution, $\mathrm{k}$-MEES is more appropriate for large scale WSNs in which network-wide communications are costly. In most scenarios, $\mathrm{k}=1$ can satisfy most application requirements that in most circumstances sensors can make decisions locally. MEES with high $\mathrm{k}$ is costly as well.

\section{Gaussian data distribution}

In this set of experiments we study k-MEES in Gaussian data distribution. Examples of the original and 1-MEES are depicted in Fig. 7 and Fig. 8 respectively. Fig. 9 plots $\left[\min \left\{\bar{\zeta}_{N r(u)}\right\}, \max \left\{\bar{\zeta}_{N r(u)}\right\}\right]$ under different $\mathrm{k}$ for k-MEES. From Fig. 9 we can find that 1-MEES presents a similar $\left[\min \left\{\bar{\zeta}_{N r(u)}\right\}, \max \left\{\bar{\zeta}_{N r(u)}\right\}\right]$ of that of uniform distribution. 2-MEES shrinks the bound to $[0.45,0.55]$ which is much tighter than $[0.39,0.61]$ in uniform scenario. And 3-MEES make a further tighter bound of $[0.48,0.52]$ which is similar to the performance of 4-MEES in uniform cases. $\mathrm{k}>4$ in Gaussion distribution can bound the measurement to a range of no more than $1 \%$. The energy cost of k-MEES and naive approaches (not shown) is similar to that of uniform distribution. Since the costs in all distributions are similar, we do not show the data in further studies due to space limitation.

From this set of experiments we can state that K-MEES is more appropriate in Gaussian distribution than that in uniform distribution. This is because Gaussian distribution has more bursty data. Only a small portion of nodes have the largely deviated sensing measurements. Such bursty data are more likely to be offset by nearby measurements. We therefore suggest a relatively high $\mathrm{k}$, for example $\mathrm{k}=2$ or $\mathrm{k}=3$, which can result in a much tighter bound with moderate cost.

\section{CONCLusion ANd Future Work}

In this paper, we investigate energy efficient design for mission critical applications. In many of these applications, the rare alerts and triggered according to aggregated sensing values such as SUM or AVERAGE from certain sets of sensor nodes. In tradition approaches, all values have to be reported to certain aggregation nodes (e.g., sinks or actuators) to make decisions. In large-scale and highly dynamic environments, however, the cost can be prohibitively high. This motivates us to design energy efficient schemes to detect critical events.

To address the need, we propose a fully distributed decisionmaking scheme so that network-wide communications are largely avoided. Our proposed MEES scheme intelligently leverages the nature that WSNs are cooperative computing environment. When every node simply does its part of job, certain global network behaviors will be presented. Theoretic analysis guarantees the correctness of MEES in the sense of a zero event-capture missing rate. Simulation experiments show that the global network property can be tightly bounded by exchanging information locally. Experiment results show that the performance of MEES highly relies on the sensory data distributions. It is more appropriate for bursty data distributions such as Gaussian distributions and Poission distributions.
In these settings 1-hop or 2-hop neighbor information is sufficient in most cases. For other distributions such as uniform distribution, distant neighbor information is needed to tightly bound the sensory measurement.

We plan to conduct our future work along following directions. First, more data distributions, such as exponential and geometric distributions, will be studied. Moreover, in real environments sensory data at nearby nodes may be spatially correlated. We are interested in effective and efficient schemes in such scenarios. Second, we assume that the critical events are threshold-based, which may not be true in practice. For example, traffic congestions may happen when vehicle distribution follows certain patterns, instead of the total volume on the road. How to effectively detect such events in a fully distributed manner is also important.

\section{ACKNOWLEDGEMENTS}

This research was supported in part by Hong Kong RGC Grants HKBU 1/05C and HKUST617908, the Key Project of China NSFC Grant 60533110, the National Basic Research Program of China (973 Program) under Grant No. 2006 CB303000 and the HKUST Nansha Research Fund.

\section{REFERENCES}

[1] R. Szewczyk, A. M. Mainwaring, J. Polastre, J. Anderson, and D. E. Culler, "An analysis of a large scale habitat monitoring application," in Proc. of ACM SenSys, 2004.

[2] I. F. Akyildiz, W. Su, Y. Sankarasubramaniam, and E. Cayirci, "A survey on sensor networks," IEEE Communications Magazine, vol. 40, no. 8 , pp. 102-114, 2002.

[3] S. Madden, M. J. Franklin, and J. M. Hellerstein, "Tag: a tiny aggregation service for ad-hoc sensor networks," in Proc. of OSDI, 2002.

[4] D. Kempe, A. Dobra, and J. Gehrke, "Gossip-based computation of aggregation information," in Proc. of IEEE FOCS, 2003.

[5] J. Considine, F. Li, G. Kollios, and J. Byers, "Approximate aggregation techniques for sensor databases," in Proc. of ICDE, 2004.

[6] S. Nath, P. B. Gibbons, S. Seshan, and Z. R. Anderson, "Synopsis diffusion for robust aggregation in sensor networks," in Proc. of ACM SenSys, 2004.

[7] S. Boyd, A. Ghosh, B. Prabhakar, and D. Shah, "Gossip algorithms: Design, analysis, and applications," in Proc. of INFOCOM, 2005.

[8] Y. Yao and J. E. Gehrke, "The cougar approach to in-network query processing in sensor networks," in Proc. of ACM SIGMOD, 2002.

[9] J. M. H. S. Madden, M. J. Franklin and W. Hong, "Tinydb: An acqusitional query processing system for sensor networks," in Proc. of ACM Transactions on Database Systems, 2005.

[10] S. B. L. Xiao and S. Lall, "A scheme for robust distributed sensor fusion based on average consensus," in Proc. of IPSN, 2005.

[11] A. D. S. A. G. Dimakis and m. J. Wainwright, "Geographic gossip: Efficient aggregation for sensor networks," in Proc. of IPSN, 2006.

[12] C. Intanagonwiwat, R. Govindan, D. Estrin, and F. Heidemann, J.Silva, "Directed diffusion for wireless sensor networking," IEEE Transaction on Networking, vol. 11, no. 1, pp. 2-16, 2003.

[13] H. Luo, F. Ye, J. Cheng, S. Lu, and L. Zhang, "Ttdd: Two-tier data dissemination in large-scale wireless sensor networks," Wireless Networks, vol. 11, pp. 161-175, 2005.

[14] B. Karp and H. T. Kung, "Gpsr: Greedy perimeter stateless routing for wireless networks," Mobile Computing and Networking, pp. 243-254, 2000.

[15] F. Ye, G. Zhong, S. Lu, and L. Zhang, "Grab: A robust data delivery protocol for large scale sensor networks," in Proc. of IPSN, 2003.

[16] D. Braginsky and D. Estrin, "Rumor routing algorithm for sensor networks," in Proc. of ACM MobiCom, vol. 2, 2002, pp. 562-567. 